Chapter 6

\title{
Neuromusculoskeletal Rehabilitation of Cerebral Palsy Using SEMLARASS
}

\author{
Deepak Sharan \\ Additional information is available at the end of the chapter \\ http://dx.doi.org/10.5772/57957
}

\section{Introduction}

The primary aim of motor treatment of Cerebral Palsy (CP) is to control and correct spasticity, abnormal movement patterns and lever arm dysfunction, since these problems directly affect the activities of daily living, ability to walk and developmental milestones.

\subsection{Disabilities in $\mathrm{CP}$}

\subsubsection{Primary disabilities}

Primary disabilities are due to the direct effect from the brain lesion, and includes spasticity, delayed milestones, sensory problems, cognitive impairment, imbalance, lack of muscle strength, abnormal movements, etc. The injury to the Central Nervous System affects critical inputs to the reticulospinal and corticospinal tract, which in turn affects the motor units and results in abnormal control and weakness. Loss of descending inhibitory input through the reticulospinal tract and other system increases the excitability of gama and alpha neurons, producing spasticity.

\subsubsection{Secondary disabilities}

Secondary disabilities are due to the after effects of primary disabilities. These include contractures, lever arm dysfunctions and scoliosis. The contractures are the after effects of spasticity. Common contractures in $\mathrm{CP}$ are hip flexion (psoas), knee flexion (hamstring) and equinus ankle (gastrocnemius) in the lower extremities, and elbow flexion (biceps and pronator) and wrist flexion (forearm flexors) contractures in the upper extremities. The concept of lever arm dysfunction was proposed by Dr. James Gage. Lever is a rigid structure that 
transmits and modifies force or motion when forces are applied at one point and is able to rotate about another. In the human body, joints function as levers to transmit forces. Lever arm (also known as a moment arm) is the perpendicular distance from the line of application of a force to the axis of joint rotation. Lever arm dysfunction is the disruption in the moment generation of a muscle joint complex because of an ineffective lever or moment arm despite normal muscle force. The types of lever arm dysfunction are: Short lever-arm (coxa valga), Flexible lever-arm (pes valgus), Malrotated lever-arm (external tibial torsion), An abnormal pivot or action point (hip subluxation or dislocation), and/or Positional lever-arm dysfunction (crouch gait).

Positional lever-arm dysfunction (crouch gait). The result of lever arm dysfunction is functional weakness and decreased power generation, due to abnormal direction of pull of muscles (Gage JR, Novacheck TF, 2001; Novacheck TF, Gage JR, 2007)

Children with $\mathrm{CP}$ are unable to perform sufficient movement to adequately stretch their muscles, particularly when those muscles are spastic. The stretch is the stimulus that facilitates muscular growth. The growing bones often become plastically deformed or twisted because of the uneven/abnormal muscle forces present during growth in a child with $\mathrm{CP}$ and the spastic muscles cause joint displacement by pulling on the bones. This leads to lever arm dysfunction in the femur as increased femoral anteversion, in the tibia as external tibial torsion and in the heel as valgus deformity. Another important lever arm dysfunction is hip joint dislocation or subluxation.

\subsubsection{Tertiary disabilities}

Tertiary disabilities are coping responses to primary or secondary disabilities and must be carefully identified and left alone. An example is circumduction of hip in co-spasticity of rectus femoris and hamstring.

\subsection{Progression of gait abnormalities in $\mathrm{CP}$}

The gait in an ambulant child with CP is characterised by crouched position (flexion of the hip and knees), intoeing (internal rotation of femur), and walking on the toes (equinus foot placement). This gait is extremely inefficient and result in increased energy expenditure due to the presence of simultaneous contraction of agonist and antagonist muscles. If the child continues walking in the presence of contractures and lever arm dysfunction the inevitable result is over lengthening of tendons (e.g., calcaneus deformity due to over lengthened tendoachilles) and joint decompensation, after which further ambulation becomes increasingly difficult.

\subsection{Overview of current treatment options for cerebral palsy and their limitations}

a. Oral Muscle Relaxants [Gracies JM, et. al, 1997; Hattab JR, 1980; Ryan DM, Blumenthal FS, 1993] Baclofen, Diazepam, Dantrolene, Tizanidine, Clonidine, Vigabatrin, Idroclimide etc. are available for the management of spasticity. The benefits of these drugs are severely limited by neurological effects such as drowsiness, daytime sedation, lassitude, exhaus- 
tion, light headedness, ataxia, confusion, dizziness, headache, insomnia, myalgia, muscle weakness and euphoria, hallucinations, nightmares, depression and dyskinesia. Other reported side effects include withdrawal symptoms, gastrointestinal disturbances such as a dry mouth, nausea, vomiting, constipation or diarrhoea.

b. Phenol \& Alcohol Injection [Kolaski K, et. al., 2008] Phenol or carbolic acid (3-7\%) and Alcohol (45-100\%) is injected directly to the spastic muscles or to the corresponding motor nerve. Phenol denatures protein and causes non-selective tissue destruction in the injected area (including coagulation of nerve and muscle) followed by Wallerian degeneration of neurons, that can occur for several weeks. Because diffusion of both is limited, the area of effective denervation extends just a few millimeters from the injection site. The duration of denervation is 3-6 months for alcohol and 4-8 months for phenol. It should be reserved only for motor nerves such as the obturator nerve and musculocutaneous nerve. Disadvantages are pain on injection requiring a general anaesthetic or heavy sedation, nonselective protein denaturation and possible permanent muscle fibrosis, dysaesthesias and motor weakness, e.g., foot drop.

c. Botulinum Toxin Type A [Jianjun L, et.al, 2013] The limitations include high expense, transient effect lasting a few weeks or months, lack of efficacy in the presence of generalised spasticity, muscle contractures or lever arm disease, and life threatening complications (permanent paralysis, respiratory paralysis and death in hundreds of children) have been reported leading to FDA issuing a warning in January 2008 against its use in CP.

d. Neurodevelopmental Therapy The policy statement from the American Academy of Cerebral Palsy \& Developmental Medicine concluded that the long-term benefits of Neurodevelopmental Therapy were marginal and/or not measurable. Except for an immediate improvement in the range of motion of joints, no other functional parameters showed any long-term improvement in a review of the available literature [Butler C, Darrah J, 2001]. A child with mild CP shows improvement with therapy, whereas the more severe cases progressively develop contractures and lever arm dysfunctions around the age of 4-7 years, after which no further improvement occurs with continuing therapy.

e. Intrathecal baclofen (ITB) therapy. A few studies have reported positive and statistically significant results for lower-extremity muscle tone. From ITB the quality of life improvement estimates were 0.27 for a bed-bound patient not in pain, to 0.5 for a bed-bound patient experiencing severe spasm-related pain. Over five years, the total discounted cost was $£ 15,400$. The cost per quality adjusted life year ranged from $£ 6,900$ to $£ 12,790$ [Sampson FC, et. al., 2002]. Several life threatening complications have been reported with ITB therapy such as complications related to pump replacement and implantation (19\%), infection (10\%), CSF leakage $(17 \%)$, catheter drawing out, disconnection or breakage (10.5\%). Drowsiness, nausea, headache, muscle weakness, light-headedness and return of pretreatment spasticity can be caused by intrathecal pump delivering an incorrect dose of baclofen [Motta F, et.al., 2007]. Intrathecal baclofen withdrawal syndrome is a very rare, potentially life-threatening complication of baclofen pump caused by an abrupt cessation of ITB [Ross JC, et.al., 2011 ]. 
f. Selective Dorsal Rhizotomy [Lundkvist A, Hagglund G, 2006; Crawford K, et. al., 1996; Mooney JF 3rd, Millis MB, 1999; Turi M, Kalen V, 2000] This is associated with adverse permanent effects (sensory disturbance, bladder dysfunction, scoliosis, lordosis, hip dislocations and foot deformities), loss of antigravity stability and worsening of motor function, and has no efficacy in patients with contractures, lever arm disease or upper limb involvement.

g. Conventional Orthopaedic Surgery. Orthopaedic surgery has been used to restore normal alignment, correcting contractures and deformities, and achieving stability by arthrodesis. Earlier it was believed that adductor spasticity was the predominant risk factor for hip dislocation, which frequently required surgical intervention [Spiegel DA, et. al., 2004]. It is now evident, after the advent of computerised gait analysis that what was called "scissoring" gait in the past is actually due to spasticity of medial hamstrings and increased femoral anteversion, in most instances [Scrutton D, et. al., 2001]. Adductor tenotomy and obturator neurectomy (a commonly performed operation) in this situation will convert an assisted ambulator into a non-ambulator, because of denervation of adductor brevis (an important hip flexor and antigravity muscle), besides producing an abduction deformity of hips. Matsuo T, et. al. [1986] compared a group of nineteen patients who had concomitant adductor tenotomy and anterior branch obturator neurectomy with a group of twenty-three patients who had only myotomy of the adductor longus and gracilis muscles. The nineteen patients in the neurectomy group had an unacceptable broad-based gait with hyperabduction of the hips. Iliopsoas lengthening or tenotomy at its insertion at lesser trochanter of femur is frequently done for a fixed flexion deformity of the hip. However, it is now known that this causes loss of power in hip flexion, and hence selective release of the psoas muscle at the pelvic brim has been recommended. [Matsuo T, et. al., 1987] The gluteus medius is a major internal rotator of the hip, and when it is spastic it can cause the objectionable in-toed gait in spastic diplegia. Steel's procedure of transfer of the insertion of the gluteus medius and minimus to the anterolateral aspect of the proximal part of the femur was effective in a few patients. But the patients in whom the procedure failed had a severe Trendelenburg limp postoperatively [Steel HH, 1980]. The correction of contractures of one joint without concomitant correction of spasticity of another joint may result in irreparable over lengthening of tendons. The commonest clinical scenario is that of a child with spastic diplegia who walks on the toes. The immediate response of many surgeons is to perform $\mathrm{Z}$ lengthening of the tendoachilles, a simple procedure that takes only a few minutes to perform, but the functional effects to the child may be devastating and permanent. Most children with spastic diplegia have hip and knee flexion contractures, and tiptoe in order to shift the center of gravity close to the body. It is extremely rare to encounter a true tendoachilles contracture in diplegics, and inappropriate lengthening of tendoachilles in the presence of hip and knee contractures inevitably leads to an unstable calcaneus deformity, which cannot be braced nor salvaged surgically. Besides, the crouch at the knee persists. The problems with Conventional Orthopaedic Surgery are: 1) Lengthening of monoarticular muscles or tendons (adductor brevis, iliopsoas, tendoachilles) leading to loss of antigravity action and severe weakness, 2) Over lengthening of tendons is common, 3) Muscle transfers (e.g., Eggers) often lead to reverse deformity, e.g., genu recurvatum, 4) It does not help the severely 
involved: quadriplegics, athetoid, dystonia, 5) Joint fusion (Grice fusion) leads to degeneration of surrounding joints, 6) Lever arm dysfunction is rarely corrected simultaneously or early enough leading to recurrence of contractures and 7) It cannot control spasticity, produce reciprocal movements to facilitate antigravity muscles, and improve functional skills and voluntary movement of the hand.

Studies have shown that the GMFCS remains unchanged or "stable" in the vast majority of children with currently available treatment modalities, including single-event multilevel surgery in bilateral spastic CP [Rutz E, et. al., 2012]. Conventional treatment for CP fails to simultaneously and effectively address spasticity, abnormal movement patterns and lever arm dysfunction, leading to a need for better rehabilitation strategies to address the functional problems in a person with $\mathrm{CP}$.

\section{SEMLARASS - A new management approach for CP}

With the help of experience of treating CP for a decade and extensive research, Dr. Deepak Sharan conceptualised Single Event Multilevel Lever Arm Restoration and Anti Spasticity Surgery (SEMLARASS) at RECOUP Neuromusculoskeletal Rehabilitation Centre, Bangalore, India in 2001.

SEMLARASS has the following components:

1. Single Event: All surgeries are completed under a single anaesthetic, requiring only one hospital admission and one period of rehabilitation,

2. Multilevel: All the affected regions and all orthopaedic deformities (soft tissue and bony) are corrected simultaneously in view of interdependence of joints,

3. Lever Arm Restoration: Simultaneous correction of lever arm dysfunctions to improve the direction of pull of muscles and to facilitate muscle strengthening postoperatively,

4. Anti Spasticity: using Orthopaedic Selective Spasticity Control Surgery (OSSCS), developed by Dr. Takashi Matsuo (Tokyo, Japan) based on the concept that multiarticular muscles, which have less antigravity activity, are hyperactive in $\mathrm{CP}$. Therefore, spasticity and athetotic movements can be controlled by releasing them selectively. The monoarticular muscles, which have antigravity activity and are responsible for maintaining an upright posture, are carefully preserved [Matsuo T, 2002].

The unique features of SEMLARASS [Sharan D, 2005] are:

a. Operating between the ages of 4 years to 6 years (preferably), to avoid joint decompensation and over lengthening of tendons that happen due to continued usage of deformed joints,

b. Minimally invasive procedures using image intensification that do not require large skin incisions and consequent risk of blood loss and infection,

c. Use of only External Fixators that do not require a second operation for removal, 
d. All bony operations done to restore deformed lever arms are extra-articular to allow for the maximum growth potential of children's bones,

e. Simultaneous lever arm restoration is essential for spasticity and contracture correction as well as to reduce chances of recurrence of deformities and repeat surgery at a later stage, and to improve the direction of pull of muscles and facilitating strengthening,

f. Tendon lengthening or transfers are avoided to reduce weakness or overcorrection, and

g. The surgery is followed by a time bound, structured and intensive physician directed rehabilitation protocol developed by Dr. Sharan.

SEMLARASS has been successfully carried out in over 1000 children from across the world. Several children have completed a follow up of 12 years and no child has had to reoperated due to recurrence of contracture or deformity.

\subsection{Recommended age for SEMLARASS}

The best functional outcomes are achieved between the age group of 4 to 6 years. The child develops a mature gait pattern by the age of 4 years and is better able to cooperate with an intensive post-operative physiotherapy programme. Once this window of opportunity is lost (usually due to reluctance of physiotherapists or physicians to let go or the insistence of the family in exploring non-operative options at any cost) and complex decompensated joint pathology has developed, the results of operation are less gratifying, though functional improvements still occur in older children and adults. Unstable lever arm disease must be operated irrespective of age if there is to be any hope of preserving ambulation.

\subsection{Advantages of SEMLARASS}

i. There is no loss of antigravity activity and weakness of the muscles because monoarticular muscles are preserved,

ii. There is no loss of sensation or sense of stereognosis,

iii. There is no increase in the occurrence of dislocations,

iv. There is little risk of recurrence of contractures and deformities with continued growth,

v. Only one hospital admission and post operative rehabilitation period,

vi. Even non ambulatory cases improve functionally and achieve a better quality of life, and

vii. More cost effective compared to other treatment options (Sharan D, 2005).

\subsection{Functional results of SEMLARASS}

The study included 314 children with $\mathrm{CP}$ with mean age $9.7 \pm 4.8$ years. The types of $\mathrm{CP}$ were spastic diplegia (58\%), spastic quadriplegia (35\%) and spastic/athetoid/dystonic quadriplegia 
(7\%). The results showed a significant improvement after a 1 year post-surgical rehabilitation. Correlation studies showed median value of Functional Mobility Scale (FMS) of 3 before surgery and 5 after surgery. Before surgery the median value of Gross Motor Functional Classification System (GMFCS) was level 4 and after surgery it was level 2. The GMFCS improved 2 levels on average. Before surgery the mean value of Pediatric QOL (PQOL) was $39.64 \pm 17.49$; after surgery the mean value was $23.11 \pm 14.02$. Before surgery median value of Manual Ability Classification System (MACS) was 3 and after surgery it was 1 . Children with severe CP (GMFCS IV and V) showed more positive correlation than mild to moderate cases. No child was wheel chair bound at the end of the rehabilitation and all the children were able to walk at least with help of a walking aid. A significant improvement was noted in their participation levels, motivation and a significant improvement in the over all quality of life. Over 50 patients have been followed up beyond 10 years and there have been no recurrences (Sharan D, 2012).

\subsection{Outcome of SEMLARASS in severe cerebral palsy (GMFCS IV AND V)}

Gross Motor Function Classification System (GMFCS) is a 5 level classification system with clinically meaningful distinctions in motor function between levels and its emphasis on selfinitiated movement with particular emphasis on sitting (trunk control) and walking. Persons with CP at GMFCS levels IV and V are non-ambulatory and at a greater risk of complications like hip subluxation/dislocation, Osteopenia/Osteoporosis and Low Energy Fractures. Prevention of these complications requires that these persons are made ambulant with or without support. However, the recommended rehabilitation strategy at present for these groups is wheel chair aided mobility leading to a "Catch 22" situation. The purpose of the study was to find out the functional outcome of SEMLARASS and rehabilitation in persons with cerebral palsy (GMFCS levels IV and V). In this study 170 children with GMFCS V\&IV were participated. Mean age of the participants was $9.68 \pm 4.77$. The follow up ranged from 1 year to 3 years (mean= 1 year). The outcome measures such as component of Gross Motor Function Measure (GMFM-88), Functional Mobility Scale (FMS), Physicians Rating Scale (PRS), Manual Ability Classification System (MACS) were used to compare the functional status of the child before and after SEMLARASS. The results showed a significant improvement in all GMFM-88 components and the values were Lying and Rolling (A); GMFM V: t-9.77 ( $\mathrm{P}<0.001)$, GMFM IV t-8.56 ( $\mathrm{P}<0.001)$, Sitting (B); GMFM V: $\mathrm{t}-20.01(\mathrm{P}<0.001), \mathrm{GMFM}$ IV: $\mathrm{t}-12.61(\mathrm{P}<0.001)$, Crawling and Kneeling $(\mathrm{C})$; GMFM V: $\mathrm{t}-22.26$ $(\mathrm{P}<0.001)$, GMFM IV: $\mathrm{t}-21.01(\mathrm{P}<0.001)$; Standing (D); GMFM V: $\mathrm{t}-20.01(\mathrm{P}<0.001)$, GMFM IV: $\mathrm{t}-22.64(\mathrm{P}<0.001)$,Walking, Running and Jumping $(\mathrm{E})$; GMFM V: $\mathrm{t}-12.71(\mathrm{P}<0.001), \mathrm{GMFM}$ IV t-15.65 $(\mathrm{P}<0.001)$, and total GMFM-88; GMFM V t-31.55 ( $<<0.001)$, GMFM IV: $\mathrm{t}-32.86$ $(\mathrm{P}<0.001)$, respectively. The result of Pre-Post PRS evaluation showed a significant improvement for both sides (Right: $t-8.60,(\mathrm{P}<0.001)$; Left: $\mathrm{t}-9.21,(\mathrm{P}<0.001)$. The improvement in the MACS (Right: t-4.05 ( $\mathrm{P}<0.001)$; Left: $t-5.74(\mathrm{P}<0.001)$ and FMS $(\mathrm{t}-5.46(\mathrm{P}<0.001)$ were also significant among both GMFCS V and IV (Sharan, 2012). 


\subsection{Complications of SEMLARASS}

A study was done to quantify the complications encountered during rehabilitation following SEMLARASS in 463 consecutive patients. The following complications were reported: Myofascial Pain Syndrome (149, 32.60\%), Prolonged Articular Stiffness beyond 4 weeks (111, $24.23 \%)$, Patellofemoral Pain Syndrome (38, 8.13\%), Osteopenia (36, 7.88\%), Meralgia Paresthetica (26, 5.69\%), Pressure Ulcers (19, 4.10\%), Hypertrophic Scar (18, 3.94\%), Low Energy Fractures $(19,4.06 \%)$, Superficial Pin Tract Infection (12,2.56\%), Wound Dehiscence $(9,1.92 \%)$, Patellar Tendinitis (8,1.71\% ), Myositis Ossificans (7,1.51\%), Complex Regional Pain Syndrome $(5,1.07 \%)$, Rickets $(3,0.6 \%)$, Osteomyelitis $(2,0.43 \%)$, Transient Common Peroneal Nerve Palsy (2, 0.43\%), Transient Axillary Nerve Palsy (2, 0.43\%), Skin Hypersensitivity $(1,0.21 \%)$, and IT Band Friction Syndrome (1, 0.21\%). There was a significant association between the anatomical distribution of abnormality and osteopenia $(\chi 2-8.01, \mathrm{p}<0.05)$. A preoperative GMFCS level IV and V was associated with a higher prevalence of complications like Osteopenia, Low Energy Fractures and Myositis Ossificans. However, none of the complications were life threatening, permanent or affecting the long term outcome of surgery. To minimise the rate of complications we recommend a structured rehabilitation protocol carried out by an experienced multidisciplinary medical team. Before the surgery, the patients, parents and care givers should be counselled regarding the prevalence of these complications, along with the available prevention and treatment options (Sharan D, 2013).

\subsection{Surgical Techniques used in SEMLARASS}

SEMLARASS differs from conventional orthopaedic surgery in the following ways:

a. Technique of surgery: conventional orthopaedic surgery relies on tendon lengthening, aponeurotic releases and tendon transfers, while SEMLARASS employs the techniques of selective spasticity control surgery, e.g., intramuscular myofascial lengthening and controlled sliding tendon lengthening. Tendon transfers are avoided in SEMLARASS.

b. Selective release of multiarticular muscles: In conventional orthopaedic surgery, a 5 year old child with spastic diplegia who walks on the toes, intoed, crouched at hips and knees, with equinovalgus would be treated by adductor tenotomy (and possibly obturator neurectomy), iliopsoas lengthening, distal hamstring lengthening or advancement (Eggers procedure), tendoachilles lengthening and Grice fusion of subtalar joint. In SEMLARASS, the procedures would be OSSCS of psoas, gracilis, medial hamstrings, gastrocnemius, femoral rotational osteotomy and medial displacement sliding calcaneal osteotomy. Hence, in conventional orthopaedic surgery several monoarticular muscles are released while in SEMLARASS only multiarticular muscles are selectively released.

c. Simultaneous release of flexor and extensor muscle groups in each joint: in SEMLARASS (except at wrists, hands and feet), while in conventional orthopaedic surgery only the flexor or the extensor muscle group is usually released.

d. Timing of surgery: conventional orthopaedic surgery is recommended in adolescence to avoid the risk of postoperative recurrence of contractures and deformities. Since SEM- 
LARASS corrects lever arm dysfunction at a much younger age the risk of recurrence is minimal.

e. Simultaneous lever arm restoration: in conventional orthopaedic surgery, lever arm restoration is left till adolescence and is often not combined with soft tissue surgery. It is frequently not addressed at all. Unlike SEMLARASS, joint fusions are often used and internal fixation (plates and screws) is used to stabilise osteotomies that requires a second operation to remove the implants.

\subsection{Assessment in SEMLARASS}

In SEMLARASS the assessment is a "bridge" between the patient, surgery, rehabilitation and prognosis. This bridge carries the patient from one phase of the treatment to the next phase. Assessments in SEMLARASS are mainly divided into: 1) Pre-operative assessment, 2) Postoperative assessment during rehabilitation and 3) Functional outcome measurement.

\subsubsection{Pre-operative assessment}

Pre operative assessment starts from first consultation with the Paediatric Orthopaedic Surgeon followed by other assessments by paediatric neurologist/developmental paediatrician, physical and occupational therapists, child psychologist, speech therapist and medical social worker.

The main objectives of the pre operative assessment are:

1. To make an accurate diagnosis, whether $\mathrm{CP}$ or other progressive neuromuscular disorder

2. To classify the type of $\mathrm{CP}$ and level of impairment

3. Screening any associated risk factors in the patient

4. To determine whether SEMLARASS is indicated

5. If SEMLARASS indicated, then to decide the nature of surgical procedures

6. For predicting the expected functional outcome

The following methods are used at RECOUP for pre-operative assessment:

1. Detailed history,

2. General assessment,

3. Assessment of sensory deficit and function,

4. Neurological assessment,

5. Musculoskeletal assessment, and

6. Instrumented gait analysis 


\subsubsection{Musculoskeletal assessment and surgical prescription}

The primary objective of the musculoskeletal assessment is to assess the musculoskeletal problems in the extremities and prescribe the appropriate surgical procedures. The assessment includes measurement of spasticity along with associated fixed deformities and lever arm dysfunctions.

\begin{tabular}{ll}
\hline Assessment & Surgical Prescription \\
\hline Staheli test (hip flexion deformity) & OSSCS psoas \\
\hline Duncan Ely's test (rectus femoris contracture) & OSSCS rectus femoris \\
\hline Adductor test (hip adductor contracture) & OSSCS gracilis, distal adductor magnus \\
\hline Popliteal angle (hamstring contracture) & OSSCS distal and/or proximal hamstring \\
\hline Silfverskiold test (gastrocnemius contracture) & OSSCS gastrocnemius \\
\hline Craig test (increased femoral anteversion) & Distal femoral derotation osteotomy \\
\hline Hip subluxation or dislocation & Proximal femoral varus derotation osteotomy \\
\hline Hip dislocation with dysplastic (shallow) acetabulum & Proximal femoral varus derotation osteotomy and \\
\hline Tibial torsion & tectoplasty \\
\hline Hind food valgus & Proximal tibial derotation osteotomy \\
\hline Plano-valgus foot with midfoot break & Medial displacement calcaneal osteotomy \\
\hline
\end{tabular}

Table 1. Surgical prescription based on pre-operative assessment

\subsubsection{Assessment during postoperative rehabilitation}

Purpose of the assessment:

1. To analyse the progress from one phase to another

2. To assess effectiveness of the treatment interventions

3. To determine the need to modify the interventions and

4. To judge the prognosis during the rehabilitation.

The main assessment methods used are:

1. Goniometry for hip, knee and ankle range of motion

2. Muscle power, spasticity and/or deformity

3. Soft tissue integrity

4. Balance and antigravity control

5. Mobility status and 
6. Over all functional status.

This periodic assessment is usually performed by the Paediatric Orthopaedics Surgeon, who makes immediate changes in the treatment protocol in discussion with the rehabilitation team during the rehabilitation to ensure the optimal functional outcome.

\subsubsection{Functional outcome measurement}

The functional outcome measurement are performed one day before the surgery, $3^{\text {rd }}$ postoperative month, $6^{\text {th }}$ month, 1 year and after 2 years. The objectives of this assessment is: 1 ) to find out the overall outcome of SEMLARASS, 2) to compare the effect of SEMLARASS in different types of CP (e.g., based on GMFCS and MACS), 3) to document the results and publish research studies related to SEMLARASS.

At RECOUP, the following outcome measures are commonly used:

1. Gross Motor Functional Measure (GMFM),

2. Modified Ashworth Scale,

3. The Amsterdam Gait Classification,

4. Melbourne Assessment of Unilateral Upper Limb Function and House Classification for upper extremity use,

5. Functional Mobility Scale (FMS),

6. Pediatric Outcomes Data Collection Instrument (PODCI),

7. The Fahn-Marsden dystonia scales, and

8. Pediatric Quality of Life Inventory (PEDS QL)

\subsection{Post-SEMLARASS rehabilitation}

A successful functional outcome after SEMLARASS requires intensive rehabilitation for atleast 2 years after the operation. Children and their parents need to be well prepared before the operation, as well as strongly motivated after the operation in order to endure the prolonged rehabilitation process. A Paediatric Physiotherapist is designated as the programme coordinator.

The entire Post-SEMLARASS Rehabilitation is divided into four phases:

a. Non-ambulatory phase: 0-2 weeks

b. Weight bearing phase: 2-4 weeks

c. Ambulatory phase: 1-6 months

d. Maintenance phase: 6-24 months 


\begin{tabular}{|c|c|c|c|c|}
\hline Stages & Time Period & Treatment Choice & Intervention & Outcome Measure \\
\hline Phase 1 & $0-2$ weeks & Soft Tissue Techniques & Oedema Control, Scar Tissue & \multirow{12}{*}{$\begin{array}{l}\text { MMT, } \\
\text { ROM }\end{array}$} \\
\hline \multicolumn{2}{|l|}{ Non Ambulatory } & & Mobilisation, MFR, Stretching & \\
\hline \multirow[t]{10}{*}{ Phase } & & Pain Management & Wax Bath, IFT,TENS, UST, Desensitisation, & \\
\hline & & Techniques & PRT, MTrP Therapy (Cross Friction & \\
\hline & & & Massage, Ischaemic Compression), & \\
\hline & & & Relaxation Exercises, CBT, Imagery, & \\
\hline & & & Breathing Exercises & \\
\hline & & Joint Mobilisation & Mulligan MWM: Hip, Knee, Ankle & \\
\hline & & Techniques & & \\
\hline & & Basic Strengthening & FES, Isometric Exercises, PRE with & \\
\hline & & Exercises & Theraband, MET, PNF, Aquatic Therapy & \\
\hline & & Balance Training & Ball Exercises & \\
\hline \multirow{12}{*}{$\begin{array}{l}\text { Phase II } \\
\text { Weight Bearing } \\
\text { Phase }\end{array}$} & \multirow{12}{*}{ 2-4 weeks } & Advanced & FES, PRE with Weight Cuffs, Core & \multirow{12}{*}{$\begin{array}{l}\text { MMT, } \\
\text { ROM }\end{array}$} \\
\hline & & \multirow[t]{3}{*}{ Strengthening Exercises } & Stabilisation, Hippotherapy, Aquatic & \\
\hline & & & Therapy, PNF, Ball Exercises, EMG & \\
\hline & & & Biofeedback & \\
\hline & & Advanced Balance & Ball Exercises, Exercises on Balance & \\
\hline & & \multirow[t]{3}{*}{ Training } & Board/ Wobble Board/Trampoline, Anti & \\
\hline & & & Gravity Muscle Strengthening, Virtual & \\
\hline & & & Reality Based Therapy & \\
\hline & & Mobility Exercises & Mat Activities, Static Cycling & \\
\hline & & \multirow[t]{3}{*}{ Gait Training } & Body Weight Supported Treadmill & \\
\hline & & & Training, Parallel Bar Walking (with & \\
\hline & & & Assistive Devices) & \\
\hline \multirow{11}{*}{$\begin{array}{l}\text { Phase III } \\
\text { Ambulatory } \\
\text { Phase }\end{array}$} & \multirow[t]{11}{*}{$1-6$ Months } & Advanced Balance & Ball Exercise, Balance Board Training & \multirow{11}{*}{$\begin{array}{c}\text { MMT } \\
\text { GMFCS } \\
\text { GMFM } \\
\text { FMS } \\
\text { PedsQL (Child \& } \\
\text { Parent Report), } \\
\text { MACS } \\
\text { The Amsterdam Gait }\end{array}$} \\
\hline & & \multirow[t]{3}{*}{ Training } & with/without Assistive Devices, Dynamic & \\
\hline & & & Mobility Exercises, Virtual Reality Based & \\
\hline & & & Therapy & \\
\hline & & Advanced & Core Stabilisation, PNF, Mat Activities, & \\
\hline & & Strengthening Exercises & EMG Biofeedback, Hippotherapy, Yoga, & \\
\hline & & & Tai Chi & \\
\hline & & \multicolumn{2}{|l|}{ Gait Training } & \\
\hline & & Sensory Re-education & Sensory Integration & \\
\hline & & Upper Limb & MFR, Stretches, CIMT, Fine Motor & \\
\hline & & Rehabilitation & Activities, Virtual Reality Based Therapy & \\
\hline Phase IV & $6-24$ & Gross Motor Functions & Cycling, Running on Treadmill, Uphill & GMFCS \\
\hline Maintenance & Months & and Fine Motor & Running, Threading a Needle, Painting, & GMFM \\
\hline Phase & & Activities & Drawing, Shoelace tying, Writing & FMS \\
\hline
\end{tabular}




\begin{tabular}{|c|c|c|c|c|}
\hline \multirow[t]{7}{*}{ Stages } & Time Period & Treatment Choice & Intervention & Outcome Measure \\
\hline & & Sporting Activities & Badminton, Basketball and Football & MMT \\
\hline & & Community Based & Community Walking & PedsQL (Child \& \\
\hline & & Rehabilitation & & Parent Report) \\
\hline & & & & PODCl \\
\hline & & & & The Amsterdam Gait \\
\hline & & & & Classification \\
\hline
\end{tabular}

Table 2. Post SEMLARASS Rehabilitation Protocol. SEMLARASS Post-Surgical Rehabilitation Intervention Pathway

\section{Abbreviations:}

PRT - Positional Release Techniques

TrP - Trigger Point Therapy

MFR - Myofascial Release

MMT - Manual Muscle Testing

ROM - Range of Motion, measured by a goniometer

MWM - Mobilisation with Movement

CBT - Cognitive Behavioural Therapy

IFT - Interferential Therapy

TENS - Transcutaneous Electrical Nerve Stimulation

UST - Ultrasonic Therapy

FES - Functional Electrical Stimulation

PRE - Progressive Resistive Exercises

MET - Muscle Energy Techniques

PNF - Proprioceptive Neuromuscular Facilitation

EMG - Electromyograph

MACS - Manual Ability Classification System

GMFCS - Gross Motor Function Classification System

GMFM - Gross Motor Function Measure

PedsQL - Paediatric Quality of Life Inventory

PODCI - Paediatric Outcome Disability Classification Index

PRS - Physician Rating Scale

FMS - Functional Mobility Scale 
CHQ - Child Health Questionnaire

CIMT - Constraint Induced Movement Therapy

\subsection{Prognosis following SEMLARASS}

The DEEPAK SHARAN'S Prognostic Score for CP is currently undergoing validation studies. The following factors have been found to determine the final functional results following SEMLARASS:

- Dysfunction of lever arm in the lower extremities

- Epileptic episodes in last two years

- Excessively lengthened tendons (e.g., due to previous Botulinum Toxin injections or Tendon lengthening surgery)

- Post operative complications in relation with severity and its duration

- Abnormalities of bone mineralisation

- Known cognitive deficits

- Sensory problems

- Hand function

- Age at the time of surgery

- Rehabilitation in terms of intensity and quality

- Achieved level of gross motor function

- Neurosurgery in the past

- Socioeconomic background

\section{Representative case studies}

\subsection{Case study 1}

\section{Prop Status}

$\mathrm{H}$, a 7 year old boy with spastic diplegia could barely take few steps when held by an adult with severe crouching at knee and toe walking. The GMFCS level was 4 .

\section{Treatment}

SEMLARASS, with OSSCS Psoas, proximal and distal Semimembranosus, Semitendinosus, Gastrocnemius, along with bilateral femoral external rotation osteotomies followed by 6 months of post operative rehabilitation. 


\section{Current functional status}

At a follow up of 6 years, he was walking and performing all the activities of daily living independently. Now he carries his bag to school by himself, climbs stairs and also participates in running, football and cycling. The current GMFCS level was 1.
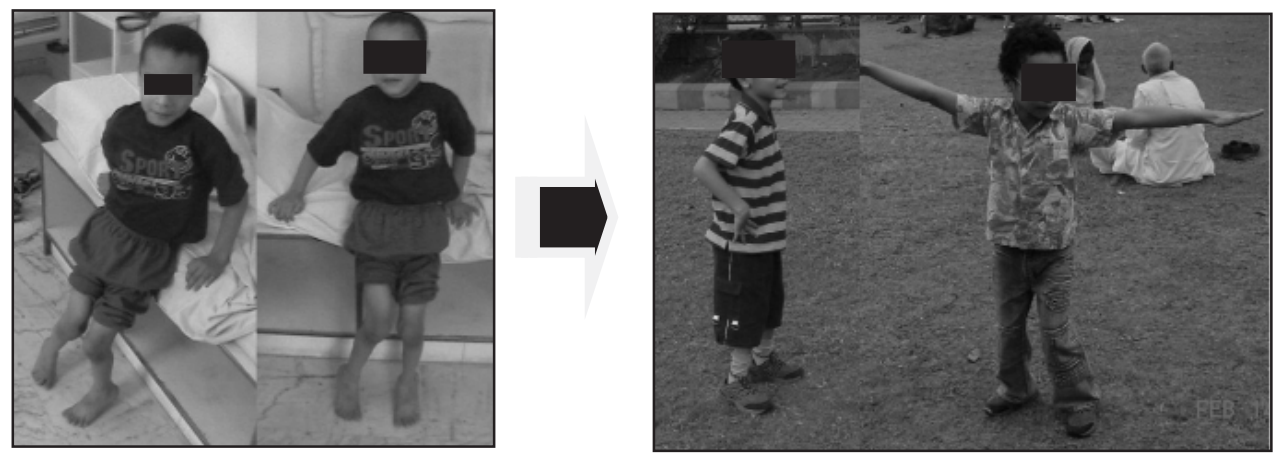

"Our son, born with Spastic Diplegia, till the age of five could only walk on toes with constant support and now after the surgery and aggressive rehabilitation at RECOUP started walking independently with lot of confidence and enthusiasm." Parents of $\mathbf{H}$

Figure 1. Before and after SEMLARASS

\subsection{Case study 2}

\section{Preop status}

S, a 10 year boy, was a known case of spastic diplegia. He was an independent ambulator with severe crouching at knee, intoeing and toe walking, with frequent falls. The GMFCS level was 2 .

\section{Treatment}

SEMLARASS, with OSSCS Psoas, distal Semimembranosus, Semitendinosus, Gastrocnemius, along with bilateral femoral external rotation osteotomies followed by 3 months of post operative rehabilitation.

\section{Current functional status}

At a follow up of 5 years, he was walking independently with a near normal gait and doing all the activities of daily living himself. Now he can sprint and play sports at a competitive level. The current GMFCS level was 1. 


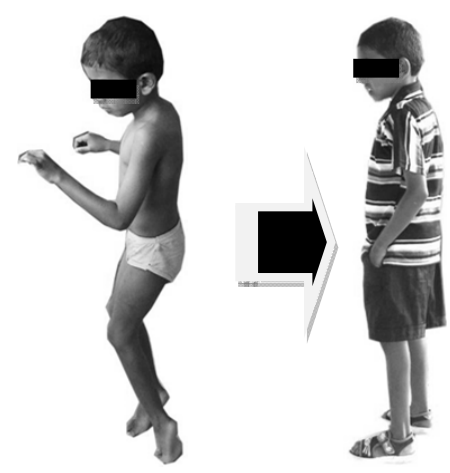

"My child made quick improvements following SEMLARASS. Now, he walks and runs independently. When he first at-
tained physical stability after the surgery he would just run around nonstop to express his joy. He now calls himself Mo-
han Lal (after the famous Malayalam Cine Star) since he believes he is now a different person altogether. We are
thankful to the RECOUP team for giving our child a life which we once thought impossible."Parents of S

Figure 2. Before and after SEMLARASS

\subsection{Case study 3}

\section{Preop status}

A, was a 12 year old girl and could barely take a few steps with extremely crouched knee with the support of an adult (GMFCS level 4). She was studying in a normal school but had to be carried by her parents to and back from the school, and could not participate in any outdoor activities.

\section{Treatment}

SEMLARASS, with OSSCS Psoas, proximal and distal Semimembranosus, Semitendinosus, Biceps Femoris, Gastrocnemius, along with bilateral femoral external rotation osteotomies, tibial internal rotation osteotomies and medial displacement calcaneal osteotomies, followed by 8 months of post operative rehabilitation.

\section{Current functional status}

At 5 year follow up, she was walking independently, doing all the activities of daily living herself, travelling to school by school bus without any assistance. The current GMFCS level was 2. 

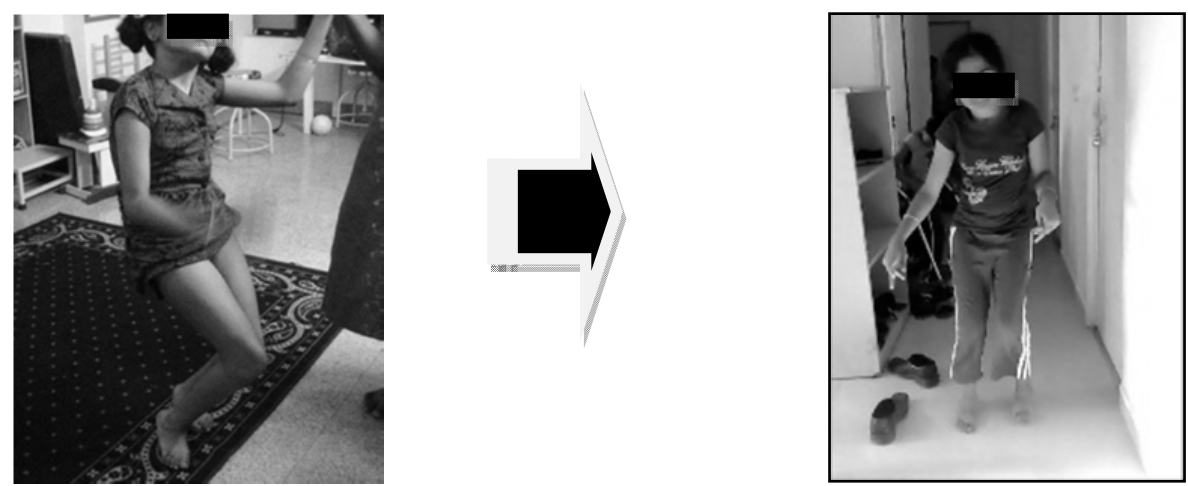

"I was 100 percent confident that, I would be able to walk independently. Finally I reached that goal. This is a rare and unexpected experience in life. Now I am enjoying my life with my friends and no barrier in front me. "A

Figure 3. Before and after SEMLARASS

\subsection{Case study 4}

\section{Preop status}

A, was a 19 year old girl with spastic athetoid hemiplegia (GMFCS level 2) with severe and disfiguring upper limb deformities, including swan neck deformities of all fingers. She had undergone failed tendon transfers in the forearm and wrist earlier.

\section{Treatment}

OSSCS right forearm flexors and pronators and hand intrinsics, distal semimembranosus, gastrocnemius, tibialis posterior and external rotation tibial osteotomy, followed by 3 months of post operative rehabilitation.

\section{Current functional status}

At a follow up of 8 years she had a cosmetically acceptable and functional upper extremity and was an independent community ambulator (GMFCS level 1).

\subsection{Case study 5}

\section{Preop status}

A, an 11 year old boy was diagnosed to have cerebral palsy (spastic quadriplegia, GMFCS level 5), was always carried by his father. He had not attained sitting balance and had severe spasticity and contractures in all 4 extremities.

\section{Treatment}

SEMLARASS, with OSSCS Psoas, Gracilis, proximal and distal Semimembranosus, Semitendinosus, Biceps Femoris, Gastrocnemius, along with bilateral femoral external rotation 

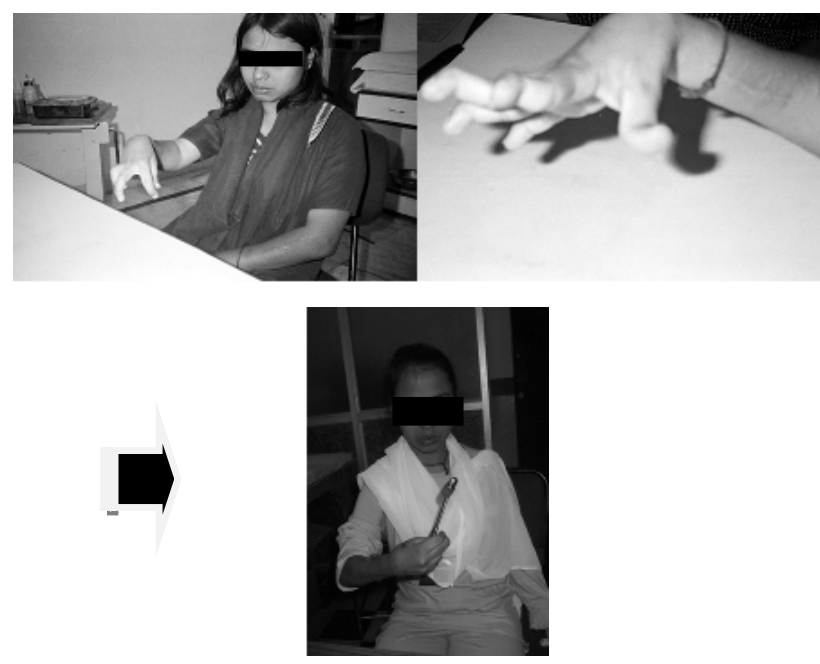

Figure 4. Before and after SEMLARASS

osteotomies, tibial internal rotation osteotomies and medial displacement calcaneal osteotomies, followed by 4 months of post operative rehabilitation.

\section{Current functional status}

At a follow up of 6 years, he was walking in the community with help of elbow crutches and a few steps independently and doing all the activities of daily living herself. Now he was studying in normal school and walked to school himself. The current GMFCS level was 2.
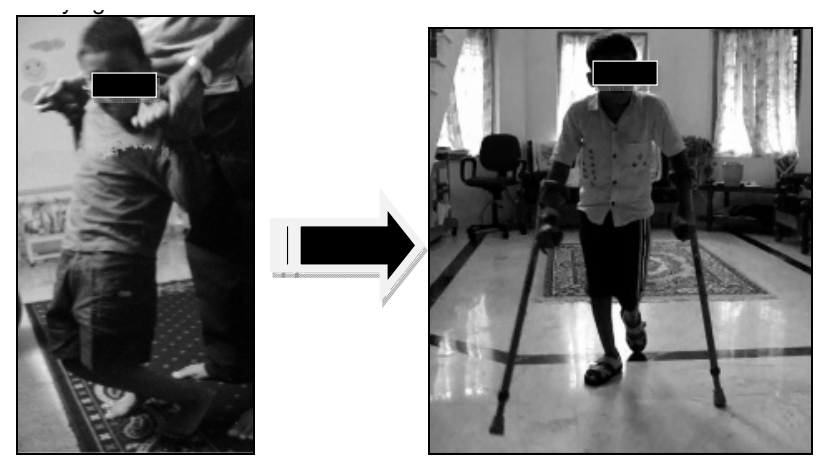

"While critically analysing the progress accomplished by my son through SEMLARASS I would like to mention that the improvement shown by him was dramatic and beyond my expectations. Before surgery I had to carry him to the school due to marked crouching and tip toeing. Now these problems are corrected to a great extent and he is walking with just one hand support and short distances independently." Parents of A.

Figure 5. Before and after SEMLARASS 


\subsection{Case study 6}

\section{Preop status}

B, an 18 year old male and a known case of spastic quadriplegia was a non ambulator, an assisted sitter with severe spasticity in all 4 extremities (GMFCS level 5). He needed two caregivers to help him transfer.

\section{Treatment}

SEMLARASS, with OSSCS Psoas, Gracilis, proximal and distal Semimembranosus, Semitendinosus, Biceps Femoris, Gastrocnemius, along with bilateral femoral external rotation osteotomies, tibial internal rotation osteotomies and medial displacement calcaneal osteotomies, followed by 8 months of post operative rehabilitation.

\section{Current functional status}

At a follow up of 5 years, he was walking with help of single stick or one hand support and doing all the activities of daily living himself. He could stand independently for short durations and was now studying in a special school. The current GMFCS level was 3.
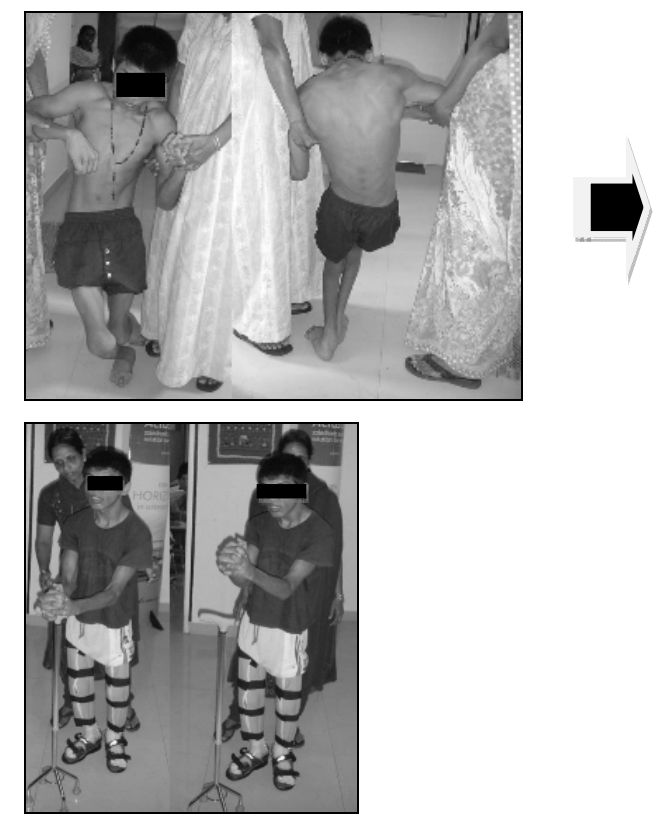

\footnotetext{
"I am extremely thankful to the staff of RECOUP for their immense efforts to help me achieve a good quality life. Now I have the ability to operate a computer and I am studying computers in my school. My future plan is to advertise the experiences and miracles in my life for helping other children with disabilities"B.
}

Figure 6. Before and after SEMLARASS 


\subsection{Case study 7}

\section{Preop status}

$\mathrm{O}$, a 10 year boy had spastic athetoid quadriplegia with generalised dystonia. He was a non ambulator and an assisted sitter with non-functional upper limbs (GMFCS level 5).

\section{Treatment}

He underwent SEMLARASS, with OSSCS Psoas, distal Semimembranosus, Semitendinosus, Gastrocnemius, along with bilateral femoral external rotation osteotomies. He also underwent OSSCS of proximal biceps femoris, triceps, distal teres major, latissimus dorsi, forearm flexors, pronators, hand intrinsics and adductor pollicis, followed by 9 months of rehabilitation.

\section{Current functional status}

At a follow up of 5 years, he could walk inside the house independently and used a single stick for walking in the community. He had good hand function on both sides and was independently going to school. The current GMFCS level was 2.
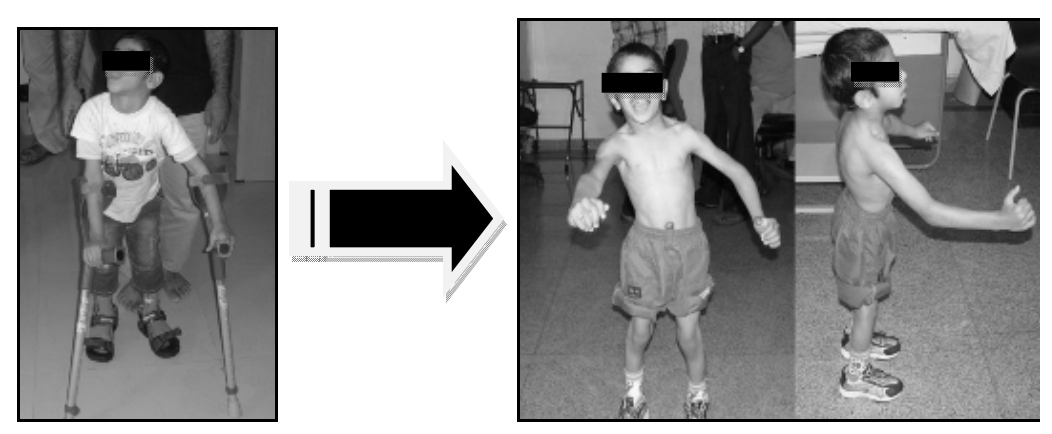

Figure 7. Before and after SEMLARASS

\subsection{Case study 8}

\section{Preop status}

S, a 10 year old girl, and a known case of spastic athetoid quadriplegia, did not have head control and was unable to sit or lie down in bed due to the severe spasticity and abnormal movements. The GMFCS level was 5.

\section{Treatment}

She underwent SEMLARASS, with OSSCS Psoas, distal Semimembranosus, Semitendinosus, Gastrocnemius, along with bilateral femoral external rotation osteotomies. She also underwent OSSCS of proximal biceps femoris, triceps, distal teres major, latissimus dorsi, forearm flexors, pronators, hand intrinsics and adductor pollicis in the second stage, followed by 9 months of rehabilitation. 


\section{Current functional status}

At a follow up of 5 years, she was walking with rollator and sitting without support. She had achieved complete head control and good hand function. Also the abnormal movements were greatly reduced. The current GMFCS level was 3.

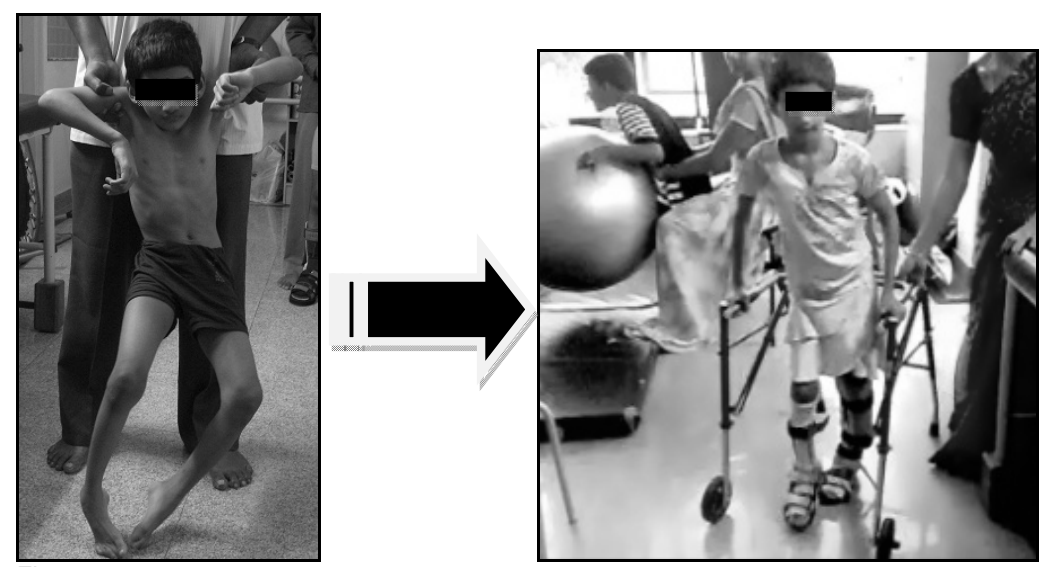

Figure 8. Before and after SEMLARASS

\section{Conclusion}

SEMLARASS is the first and only treatment modality for CP to systematically, simultaneously and effectively address all the major problems restricting the child's functional activities, i.e., spasticity or movement dysfunction and lever arm dysfunction. Currently, a well-planned and executed SEMLARASS, in the context of a multi-disciplinary team, provides the child with CP with the best hope for a dramatic, predictable and lasting functional improvement (e.g., improved GMFCS level). This is especially true of spastic quadriplegia, dystonia and athetosis that account for over two-thirds of cases of $\mathrm{CP}$, where available treatment options are extremely limited.

\section{Author details}

\section{Deepak Sharan*}

Address all correspondence to: deepak.sharan@recoup.in

Dept. of Paediatric Orthopaedics and Rehabilitation, RECOUP Neuromusculoskeletal Rehabilitation Centre, Bangalore, Karnataka, India 


\section{References}

[1] Gage JR, Novacheck TF. An update on the treatment of gait problems in cerebral palsy. J Pediatr Orthop B. 2001 Oct;10(4):265-74.

[2] Novacheck TF, Gage JR. Orthopedic management of spasticity in cerebral palsy. Childs Nerv Syst. 2007 Sep; 23(9):1015-31.

[3] Gracies JM, Nance P, Elovic E, McGuire J, Simpson DM. Traditional pharmacological treatments for spasticity. Part II: General and regional treatments. Muscle Nerve Suppl. 1997; 6: S92-120.

[4] Hattab JR. Review of European clinical trials with baclofen. In: Feldman RG, Young RR, Koella WP (eds.), Spasticity: Disordered Motor Control. Chicago: Year Book Medical Publishers 1980, pp. 71-86.

[5] Ryan DM, Blumenthal FS. Baclofen-induced dyskinesia. Arch Phys Med Rehabil. 1993 Jul; 74(7): 766-7.

[6] Kolaski K, Ajizian SJ, Passmore L, Pasutharnchat N, Koman LA, Smith BP. Safety profile of multilevel chemical denervation procedures using phenol or botulinum toxin or both in a pediatric population. Am J Phys Med Rehabil. 2008 Jul; 87(7): 556-66.

[7] Jianjun L, Shurong J, Weihong W, Yan Z, Fanyong Z, Nanling L. Botulinum toxin-A with and without rehabilitation for the treatment of spastic cerebral palsy. J Int Med Res. 2013 Jun; 41(3): 636-41.

[8] Butler C, Darrah J. Effects of neurodevelopmental treatment (NDT) for cerebral palsy: an AACPDM evidence report. Dev Med Child Neurol. 2001 Nov; 43(11): 778-90.

[9] Sampson FC, Hayward A, Evans G, Morton R, Collett B. Functional benefits and cost/benefit analysis of continuous intrathecal baclofen infusion for the management of severe spasticity. J Neurosurg. 2002 Jun; 96(6): 1052-7.

[10] Motta F, Buonaguro V, Stignani C. The use of intrathecal baclofen pump implants in children and adolescents: safety and complications in 200 consecutive cases. J Neurosurg. 2007 Jul; 107(1 Suppl): 32-5.

[11] Ross JC, Cook AM, Stewart GL, Fahy BG. Acute intrathecal baclofen withdrawal: a brief review of treatment options. Neurocrit Care. 2011 Feb; 14(1): 103-8

[12] Lundkvist A, Hagglund G. Orthopaedic surgery after selective dorsal rhizotomy. J Pediatr Orthop B 2006, 15: 244-246.

[13] Crawford K, Karol LA, Herring JA. Severe lordosis after dorsal rhizotomy. J Pediatr Orthop. 1996 May-Jun; 16(3): 336-9.. 
[14] Mooney JF 3rd, Millis MB. Spinal deformity after selective dorsal rhizotomy in patients with cerebral palsy. Clin Orthop 1999; 364: 48-52.

[15] Turi M, Kalen V. The risk of spinal deformities after selective dorsal rhizotomy. J Pediatr Orthop 2000; 20(1):104-7.

[16] Spiegel DA, Loder RT, Alley KA, Rowley S, Gutknecht S, Smith-Wright DL, Dunn ME. Spinal Deformity Following Selective Dorsal Rhizotomy; J Pediatr Orthop 2004; 24: 30-36.

[17] Scrutton D, Baird G, Smeeton N. Hip dysplasia in bilateral cerebral palsy: incidence and natural history in children aged 18 months to 5 years. Dev Med Child Neurol 2001; 43: 586-600.

[18] Matsuo T, Tada S, Hajime T. Insufficiency of the hip adductor after anterior obturator neurectomy in 42 children with cerebral palsy. J Pediatr Orthop. 1986 Nov-Dec; 6(6): 686-92.

[19] Matsuo T, Hara H, Tada S. Selective lengthening of the psoas and rectus femoris and preservation of the iliacus for flexion deformity of the hip in cerebral palsy patients. J Pediatr Orthop. 1987 Nov-Dec; 7(6): 690-8.

[20] Steel HH. Gluteus medius and minimus insertion advancement for correction of internal rotation gait in spastic cerebral palsy. J Bone Joint Surg Am. 1980 Sep; 62(6): 919-27.

[21] Rutz E, Tirosh O, Thomason P, Barg A, Graham HK. Stability of the Gross Motor Function Classification System after single-event multilevel surgery in children with cerebral palsy. Dev Med Child Neurol. 2012 Dec; 54(12): 1109-13.

[22] Matsuo T. Cerebral Palsy: Spasticity control and Orthopaedics: An Introduction to Orthopaedic Selective Spasticity Control Surgery. Soufusha, Tokyo, 2002.

[23] Sharan D. Recent advances in the management of Cerebral Palsy. Indian Journal of Pediatrics 2005; 72(11): 969-973.

[24] Sharan D. Functional Outcome of a new Single Event Multilevel Lever Arm Restoration and Anti Spasticity Surgery for Cerebral Palsy. 33 ${ }^{\text {rd }}$ Orthopaedic World Conference, Dubai, United Arab Emirates (November 28-30, 2012).

[25] Sharan D. Functional Outcome of a New Surgical Approach in Severe Cerebral Palsy (GMFCS IV and V), $4^{\text {th }}$ International Cerebral Palsy Conference, Pisa, Italy (October 10-13, 2012).

[26] Sharan D. Complications during Post-Surgical Rehabilitation Following Single Event Multilevel Surgery in Cerebral Palsy, $14^{\text {th }}$ International Society for Prosthetics and Orthotics 2013 World Congress, Hyderabad, India (February 4-7, 2013). 
